\begin{tabular}{|c|c|c|}
\hline \multirow{3}{*}{$\begin{array}{l}\text { ITC 4/47 } \\
\text { Journal of Information Technology } \\
\text { and Control } \\
\text { Vol. } 47 / \text { No. } 4 \text { / } 2018 \\
\text { pp. } 623-638 \\
\text { DOI 10.5755/j01.itc.47.4.21264 }\end{array}$} & \multicolumn{2}{|c|}{$\begin{array}{l}\text { A Method for Reverse Engineering UML Use } \\
\text { Case Model for Websites }\end{array}$} \\
\hline & Received 2018/07/17 & Accepted after revision 2018/09/27 \\
\hline & \multicolumn{2}{|c|}{ Crossef http://dx.doi.org/10.5755/j01.itc.47.4.21264 } \\
\hline
\end{tabular}

\title{
A Method for Reverse Engineering UML Use Case Model for Websites
}

\section{Lina Čeponienė, Vaidotas Drungilas, Mantas Jurgelaitis, Jonas Čeponis}

Faculty of Informatics; Kaunas University of Technology; Studentų Str. 50, LT-51368, Kaunas, Lithuania; e-mails:lina.ceponiene@ktu.lt,vaidotas.drungilas@ktu.lt,mantas.jurgelaitis@ktu.lt,jonas.ceponis@ktu.lt

Corresponding author: lina.ceponiene@ktu.lt

Problems with inadequately documented or undocumented websites could be alleviated by introducing reverse engineering of UML diagrams. In this paper, the method for reverse engineering UML use case model for websites is presented. It consists of two steps: recording user actions in the analyzed website, and then transforming the combination of recorded activity and publicly available HTML code information into UML use case model. This model consists of UML use case diagram and UML activity diagrams describing scenario of each use case. The proposed method is implemented as a Google Chrome plugin named WEB2UML. WEB2UML is able to generate UML use case and activity diagrams in XMI format, compatible with MagicDraw UML CASE tool. During experimental evaluation of the WEB2UML tool, two UML use case models were reverse engineered: one for moodle. if.ktu.lt website and another for researchgate.net website. The quality of generated models was evaluated using an anonymous questionnaire completed by 13 UML modelling experts. The results of expert evaluation are encouraging: in total, average expert evaluation score was 8,4 in a scale of ten.

KEYWORDS: UML, use case diagram, activity diagram, reverse engineering, website.

\section{Introduction}

UML is a commonly used modelling language encompassing a wide range of various diagrams for specifying different aspects of software systems. UML use case diagrams are used for expressing user requirements [15, 30]. Use case diagram supplemented by descriptions for each use case forms use case model of the system. Use case description contains a scenario, which can be represented by UML activity diagram [3]. In this paper, we analyze use case model composed of UML use case diagram and UML activity diagrams for each use case. The use case model can be used during development and while maintaining Web systems [12]. This model serves as a tool for communication inside the development team as well as between customers and develop- 
ers, because it helps to represent the system in terms of its functional usage [10].

Model Driven Engineering (MDE) [28] is an approach which strives to improve software development processes by introducing modelling into different steps of the process, such as implementation, testing, maintenance, etc. MDE employs UML models for representing complex systems and proposes transformations for software development. Apart from utilizing the potential of model transformations, MDE emphasizes the fact that modeling can help to decrease the complexity of software development tasks, as the models can be used to represent the analyzed system in different levels of abstraction [7].

However, problems of keeping UML models up to date arise, as updating the diagrams requires additional effort from developers. Websites more often than any other type of software are subject to constant updates and improvements, as they have to meet ever changing user requirements and succeed in highly competitive market [17, 21]. Website developers, facing continuous changes in requirements, tend to sacrifice the quality of website documentation and spend more resources on development of new website functionality [26]. If not properly maintained, UML models lose their practical value and can even be misleading. Finding and fixing the discrepancies between documented UML models and the present functionality of a web system, requires significant effort from development team. Likewise, the support of legacy systems, whose documentation is not available, is also a challenging task [14].

The problems with inadequately documented or undocumented systems can be alleviated by introducing reverse engineering of UML models. Reverse engineering is defined as a process of system analysis performed in order to identify the systems structure and behavior and represent them in a different form or using a higher level of abstraction [5]. Model Driven Engineering principles can be successfully applied in reverse engineering area: Model Driven Reverse Engineering (MDRE) concentrates on applying MDE strategies for developing efficient reverse engineering solutions [4]. Reverse engineered UML diagrams can help to visualize, improve understanding and provide documentation for the existing functionality of web systems [27, 32].

In this paper, a method for reverse engineering UML use case model from web systems is presented. This method enables transformation of recorded website user activity into UML use case and activity diagrams. The method consists of two main steps: recording user actions in the website, and then transforming the combination of recorded activity and HTML code information into UML use case model.

Our method is based on dynamic analysis of website usage, without analyzing the internal source code. Only directly accessible HTML code of website is analyzed together with the recorded user activity. Many existing methods for reverse engineering UML diagrams require access to the source code of system under analysis $[2,6,9,16]$. Reverse engineering UML diagrams from websites without access to source code can be useful in various situations, e.g. during analysis of undocumented legacy systems for finding out their functionality or for comparing functionality of existing public websites [1]. Furthermore, reverse engineering UML diagrams excluding analysis of source code ensures that our approach is language independent, which broadens the set of web applications that can be reverse engineered using our method. Other methods, not relying on source code analysis exist, but these methods mainly generate UML sequence, state diagrams $[13,33]$ or non-UML based visualizations [18] for websites. None of the aforementioned methods generates both use case and activity diagrams.

Our approach also employs real web application users - they can perform their usual activities in website, just providing information about their role and performed processes, and the tool records these activities for further transformation into UML use case and activity diagrams. A Google Chrome plugin as a prototype tool was implemented for evaluating the proposed method. Experiment results indicate that the tool is capable of reversing use case models for websites. Although there still are some disadvantages in method implementation, such as action naming problems in activity diagrams, experts evaluated the quality of generated models as adequate.

The rest of the paper is organized as follows. The second section analyses related work in the area of reverse engineering UML diagrams. The proposed methodology for reverse engineering UML use case and activity diagrams from recorded activity of website usage is presented in the third section. The fourth section analyses method implementation. Experiment setting and results are discussed in the fifth section. Finally, conclusions and future work are presented in the last section. 


\section{Related Work}

Reverse engineering can facilitate comprehension and decrease effort required for website maintenance, reengineering or evolution [5]. UML-based reverse engineering is quite popular as UML is commonly used as a standard for software modelling and as a tool for communication [27]. UML diagrams provide a clear notation, which can be used to construct diagrams for various aspects of systems. Reverse engineering of UML diagrams can be useful for improving understanding of program code in various areas of software development even in software development education [32]. UML diagrams are suitable for specifying system structural elements and behavior at various abstraction levels [24]. Structural UML diagrams, such as class, component or package diagrams can be used to represent static structures in terms of system parts and their relations. Furthermore, behavioral diagrams, such as activity, use case or sequence diagrams, are able to express dynamic behavior of objects in the analyzed system.

Reverse engineering methods cover both structural and behavioral UML diagrams, but in general, static structures can be reverse engineered with less difficulty than behavioral diagrams. A number of UML CASE tools, for example MagicDraw [23] and Visual Paradigm [31], already support reverse engineering of structural UML diagrams, such as class diagrams [25]. Although CASE tools provide similar functionality, a need for more effective ways of reverse engineering structural diagrams still exists. For example, in [8] a method for reverse engineering class diagrams is presented, which is more effective and flexible in terms of scalability than reverse engineering options in existing CASE tools. This method uses a set of accurate mappings for representing $\mathrm{C}++$ syntactic and semantic information in UML class diagrams.

In contrast, reverse engineering behavioral diagrams is a more complicated task, not so widely supported by UML CASE tools. Sequence diagrams can be reverse engineered in several UML CASE tools, such as Enterprise Architect [29], but the implementation of this feature is quite primitive [25]. Quite a lot of studies analyze the possibilities for reverse engineering sequence diagrams, such as [2, 9, 13, 33]. Reversing other behavioral diagrams, like activity diagrams, use case diagrams and state machines is not supported by
CASE tools, although there is also a number of studies in this area $[11,13,19]$.

There are several solutions focusing on reverse engineering of behavioral UML diagrams, particularly for websites. The reverse engineering tool PHP2XMI [2] is able to generate behavioral model of a website, expressed as an UML sequence diagram. The tool analyses PHP-based web applications' source code for inserting probes to collect dynamic information, stores and filters execution traces generated by the probes during interactive browser sessions and transforms recorded execution traces into UML sequence diagrams.

Another website-oriented approach is presented in [9]. In this approach, a tool named WARE is proposed for website reverse engineering of UML use case, sequence and class diagrams. WARE tool uses static code as an input and is capable of reverse engineering only when having access to entire source code of web application. In general, when source code is available, reverse engineering UML diagrams seems to have more possibilities for implementation, as source code is a valuable source of information $[2,6,9,16]$. Unfortunately, the source code is not always available. In addition, analysis of application without examining source code ensures that our method is language independent. It is also important for analysis of a wide range of websites implemented using different technologies. Our research concentrates on reverse engineering websites without access to internal source code and utilizing only information acquired from publicly available HTML code and recorded user activity in the website.

In situations where the source code is not available, an approach of extracting information from a working system can be used for reversing UML behavior diagrams [33]. This approach focusses on fully dynamic analysis of the system for reverse engineering of UML sequence diagrams and is intended for systems where static code analysis cannot be directly applied. The process of reverse engineering starts with collecting execution traces (sequences of method invocations) for the analyzed system. The collected traces are later merged into a Labeled Transition System (LTS), which is transformed into a sequence diagram. The idea of applying dynamic analysis for gathering execution traces of analyzed system is also employed in our proposed solution. Another approach based on collecting trace information is presented in [13]. This 
method is intended for (re)documenting API usage and uses trace information, gathered by monitoring behavior of applications, which are using the API. Afterwards, UML state machine and sequence diagrams are reverse engineered from the gathered information. The problems of merging the recorded execution traces are addressed in [22], where a method for identifying systems key behavior and generating a readable sequence diagrams is presented.

There are several studies on reverse engineering specifically use case diagrams. Some of them are based on source code analysis $[6,9,16]$, which is not applicable to our method. Others have one major disadvantage: they require effort for creating additional specifications which are used as an input for diagram generation. In [11], reverse engineering is used for generating use case models from structured textual use case specifications. In [19], event tables are used for use case model generation. In event tables, information about each event, the source of event, action and associated object must be provided for generating informative use case model. In our work, we decided to minimize required additional input, as requirement for exhaustive additional specifications compromises the whole idea of simplifying diagram extraction process.

Reverse engineering approaches, using different form of visualization (e.g. $[18,20]$ ) than UML-based approaches, provide further insight. In [18] the approach for visualization of web-based systems user interface is proposed. User interface models, which use information visualization technique ModelUI $_{\mathrm{VIZ}}$, are reverse engineered from web applications. The tool for this approach, WMUID, was implemented as a Google Chrome plugin, which uses Google Chrome API to access website elements. Both crawler and tracer mechanisms are used for data extraction. The crawler analyses user interface and identifies interactive elements, such as buttons, inputs and links. The tracer monitors and registers user interaction and navigation. Another tool, WMUIT, is used for displaying the data collected by WMUID tool. In our work, a tool similar to the tracer is also implemented as a Chrome plugin. In general, the principles of [18] are successfully applied in our research.

Our proposed method analyses information acquired by recording website usage. It does not require access to the websites source code to perform static code analysis, as dynamic analysis is enough for gathering required information. The only additional information source for our reverse engineering method is publicly available website HTML code. Based on other methods' implementations, we decided to implement usage recording tool as a browser plugin for gathering event sequences, which can then be converted to use case and activity diagrams.

\section{A Method for Reverse Engineering UML Use Case Model}

The proposed method for reverse engineering UML use case model enables dynamic analysis of web applications, in order to generate UML use case model. The method does not require access to internal source code of the application - only recorded user actions and public HTML content is used. The created use

\section{Figure 1}

General process for reverse engineering of UML use case model

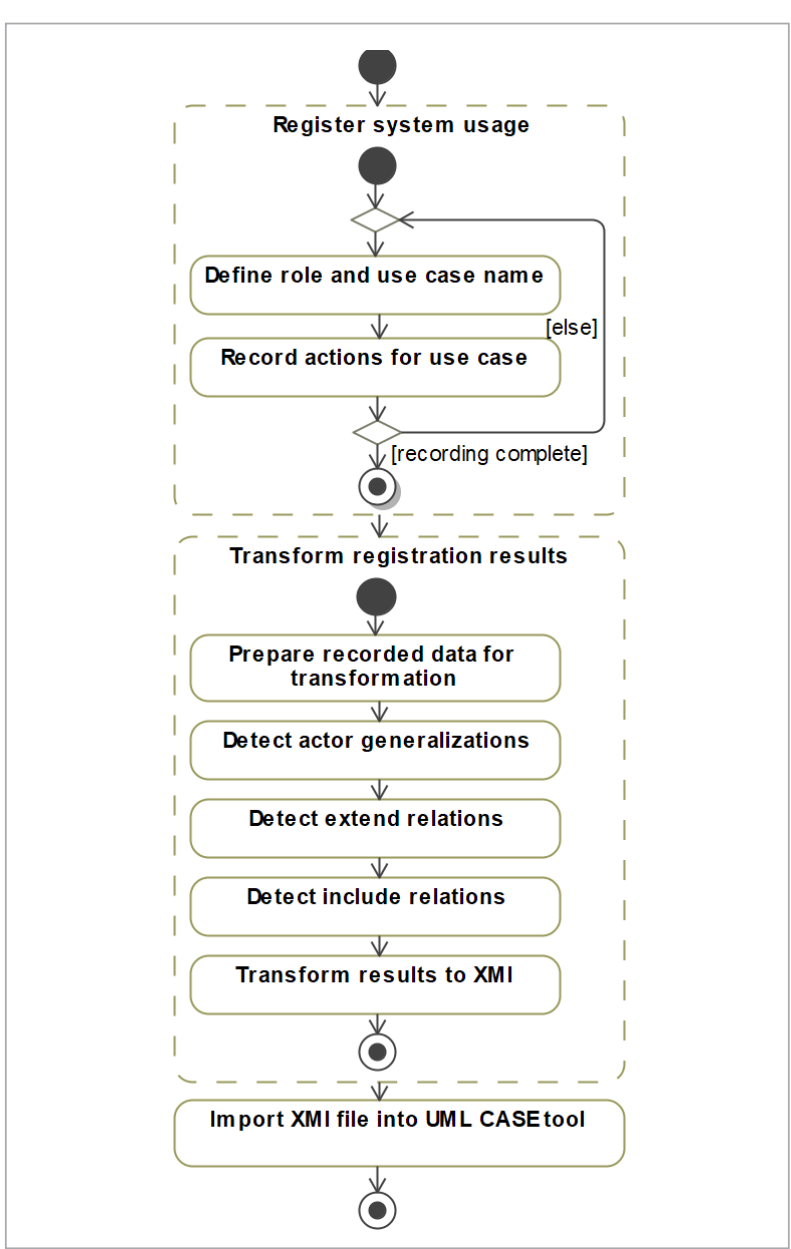


case model encompasses UML use case diagram along with specifications of each use case in a form of UML activity diagrams. Each activity diagram specifies a use case scenario with required include and extend relations between use cases. Alternative scenarios are also represented in activity diagrams, as the method is able to combine recorded scenarios for appropriate use cases. In reverse engineered use case diagram, generalizations between actors are also determined and additional actors may be created if situations arise when use case sets are overlapping. The method consists of two main steps, after which the generated UML diagrams can be imported, viewed and edited in UML CASE tool (Figure 1).

The first step of the method is to record user actions in the website under analysis. The user indicates his role and process (use case) he is going to perform in the analyzed website. Afterwards, user performs actions in the website for particular process and the tool logs the events' sequences. When the user indicates, that he has recorded actions for all required usage scenarios of the website, the second step can be performed. Initially, website usage recording results are prepared for transformation by removing actors' and use cases' duplicates. Afterwards, registered event sequences are combined and various types of appropriate rela- tions between actors and use cases are detected, such as generalizations between actors, include and extend relations between use cases, associations between actors and use cases. The results are then transformed into an XMI file which can be imported in UML CASE tool for further usage.

\section{Algorithms for Relation Detection}

In this paper, we present in detail three most important algorithms of our method - actor generalization detection, extend relation detection, and include relation detection. These algorithms make up the main group of procedures required for transforming recorded user activity into UML diagrams. The algorithms are performed sequentially, as defined in Figure 1.

The first algorithm (Figure 2) detects generalization relations between actors in use case diagram by analyzing recorded data associated with user defined roles. The roles that user specifies during activity recording are recognized as actors in our method. Each actor has a set of recorded processes, which are identified as use cases associated with this actor. The actors owned use case sets are compared, and two types of match can be detected (Figure 2, line 8): full or partial. A full match

Figure 2

Algorithm in pseudocode for detecting generalizations between actors

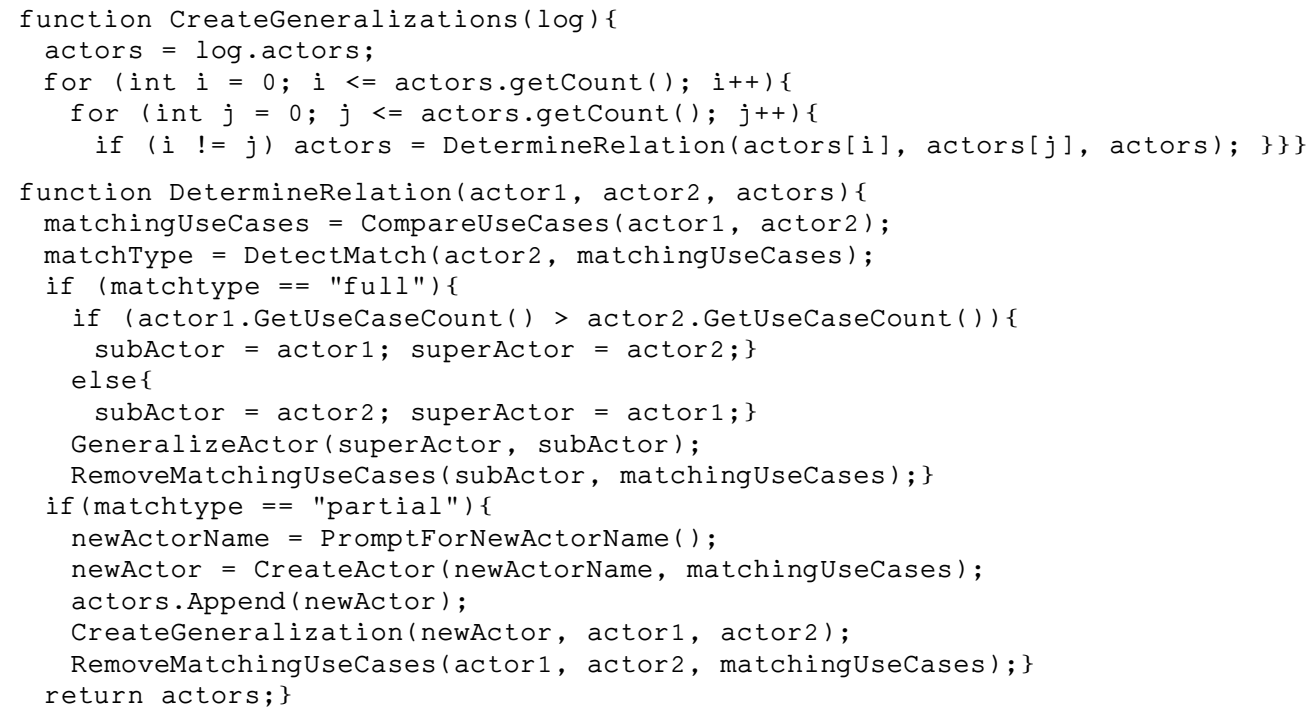


between use case sets is detected when intersection between two actors use case sets is equal to use case set of one of these actors. In that case, we have to determine subactor (actor who has more use cases) and superactor (which has less use cases) for creating generalization (Figure 2, lines 10-14), and then remove all of subactors' inherited and now redundant use cases from his set (Figure 2, line 15). Another type of match between use case sets is a partial match. Partial match occurs when intersection between actors use case sets is not empty and is not equal to use case set of one of the actors. Then our algorithm creates a new actor, which will generalize both compared actors, and prompts for a new actor name (Figure 2, lines 17-18). Use cases from the intersection of compared actor sets are associated with the new actor (Figure 2, line 19). Afterwards, a generalization is created between new actor and each of the compared actors (Figure 2, line 20). Lastly, the redundant inherited use cases are removed from compared actors sets (line 21). The comparison of use case sets is repeated until all actors are compared to each other, including the ones created during the generalization creation.

The second algorithm detects extend relations between use cases in use case diagram. It is worth mentioning that both include and extend use case relations must also be represented in activity diagrams. For each include relation between including and included use case, there must exist a reference (in the form of callBehaviorAction) in including use cases' activity diagram. Analogously, for each extend relation between extended and extending use case, in extended use cases' activity diagram, there must exist a decision node (representing the choice of performing the extending use cases activity diagram), a reference (in the form of callBehaviorAction), and a merge node.

General process of extend relation detection is presented in Figure 3, where main steps of the process are marked as arrows and numbered. The pool of user defined processes recorded during website usage (recognized as use cases) is analyzed.

In the first step, use cases with repeated names are selected. As a result of the first step, subsets are created for each group of same named use cases. In the second step, one use case subset is selected for further analysis. During the third step, the sequences of recorded actions are compared by finding matching subsequences. The sets are modified in such a way,

\section{Figure 3}

Main steps of extend relation detection

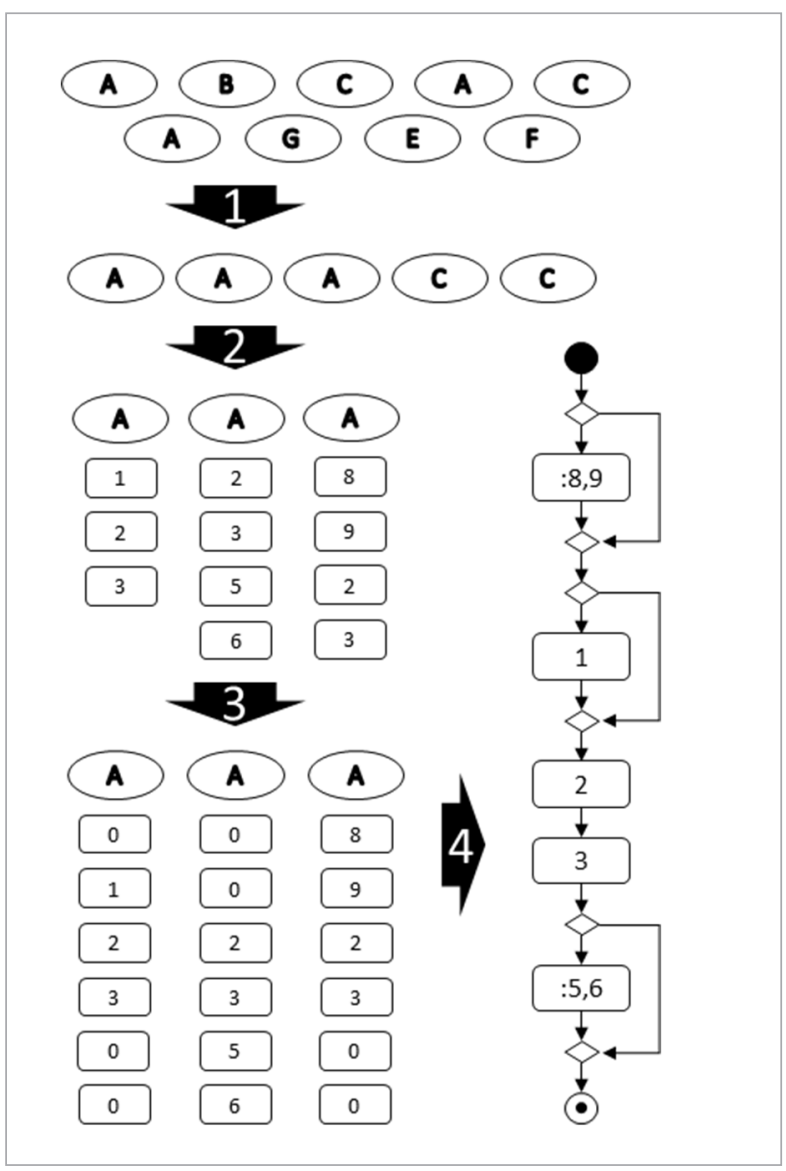

that matching sequences are positioned along the same indexes, and placeholder variables (" 0 " in Figure 3) are appended at the beginning and the end of the sequences for alignment. An example of aligned sequences is presented in Figure 3 as a result of the third step. Matching sequence in this case consists of recorded actions 2 and 3 .

In the fourth step, the new use case combining all subset use cases is created and its activity diagram is gradually generated. The required elements (e.g. actions, decision and merge nodes) are gradually appended to activity diagram and joined with control flow relations. To determine the order of appending elements, matching sequences of actions are detected. Each action of subsets' use case is processed depending on whether it exists in a range of matching elements or not. When the action exists in the range of matching actions, and we have reached the appro- 
priate place during combined activity diagram creation, new action element is created and appended to the activity diagram. Otherwise, actions that do not exist in matching range are included in a subsequence starting with non-matching action and ending when action in matching range is found or main sequence ends. Figure 4 presents a pseudo-code fragment demonstrating the processing of non-matching subsequences. If non-matching subsequence contains less actions then determined beforehand (Figure 4, line 4), its actions are appended to the activity. Decision and merge nodes are inserted, respectively, before and after the actions, joining them with control flow relations (Figure 4, lines 14-16). If non-matching subsequence contains more actions than the defined constant, a new use case is created. Use case name must be provided for creation (Figure 4, line 18) and activity diagram is also created for this use case. Actions of non-matching sequence are inserted into the new activity diagram (Figure 4, line 20). The new extending use case and combined use case are joined by extend relation (Figure 4, line 21). Combined use cases' activity diagram is appended with decision node, reference (callBehaviorAction) to the new activity diagram, and merge node (Figure 4, lines 22-24).
The third algorithm is used for detecting include relations between use cases. It is started only when all extend relations are detected and all alternative sequences are combined into respective use cases. In Figure 5, pseudocode fragment for include relation detection is presented. In the beginning, matching recorded actions' sequences longer than the defined constant (Figure 5, line 3) are detected in all use cases (Figure 5, line 4). Use cases are grouped in subsets where matching sequences were found (Figure 5, line 5). For each use case subset, a new use case (which will act as included use case) is created, whose name must be provided. Additionally, a new activity diagram for the new use case is created encompassing the matching sequence (Figure 5, line 12). The actions of matching sequence are removed from all the use cases in the subset. The new use case is then joined with the including use cases from the subset using include relation. For all subset use cases, a reference (callBehaviorAction) to the new use case is inserted instead of removed matching sequence.

The result of execution of all three presented algorithms is transformed into XMI format. This ensures that resulting diagrams can be modified and improved using CASE tool for further usage. The resulting XMI

\section{Figure 4}

Fragment of algorithm in pseudocode for extend relation detection

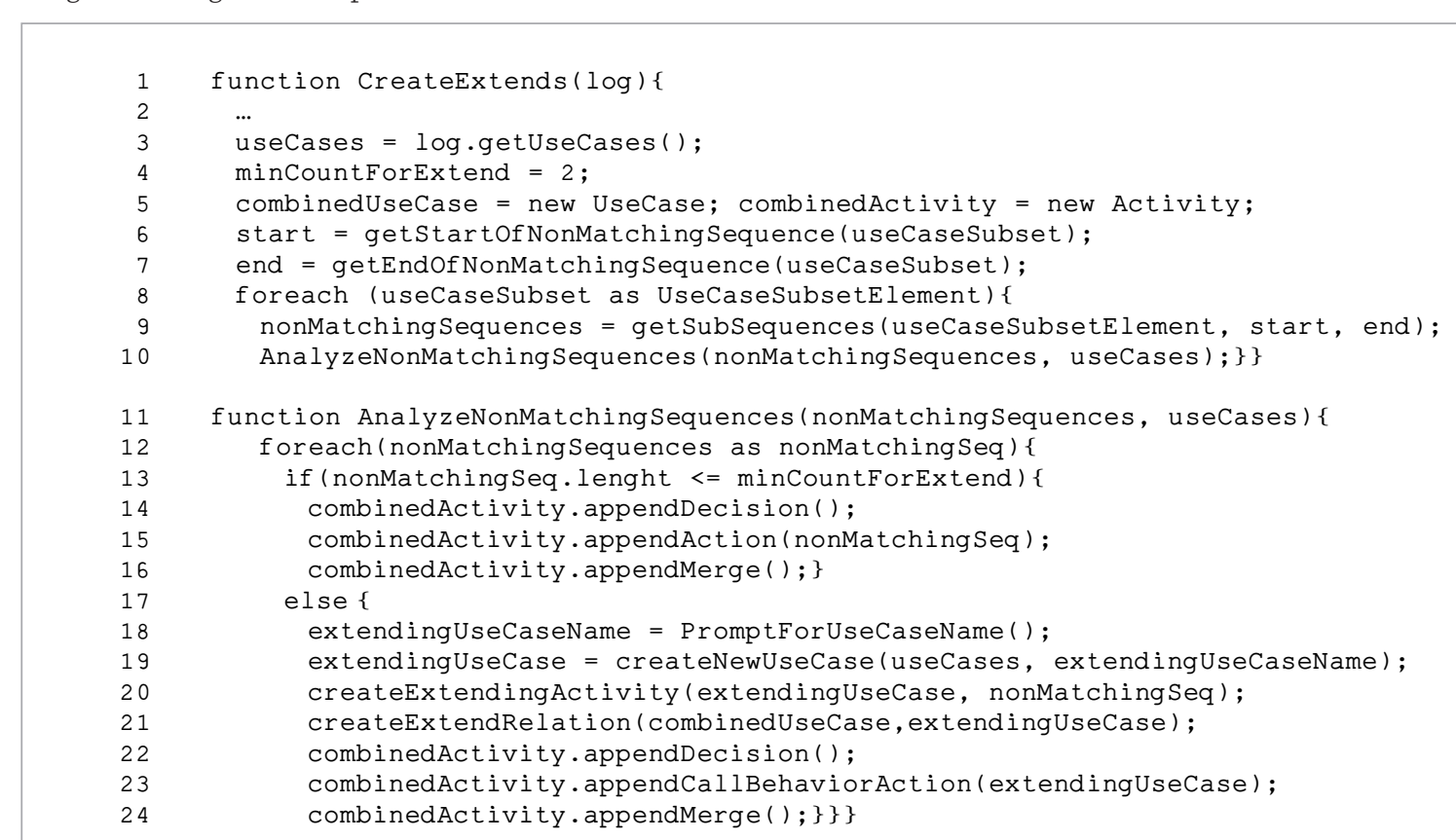


Figure 5

Algorithm in pseudocode for include relation detection

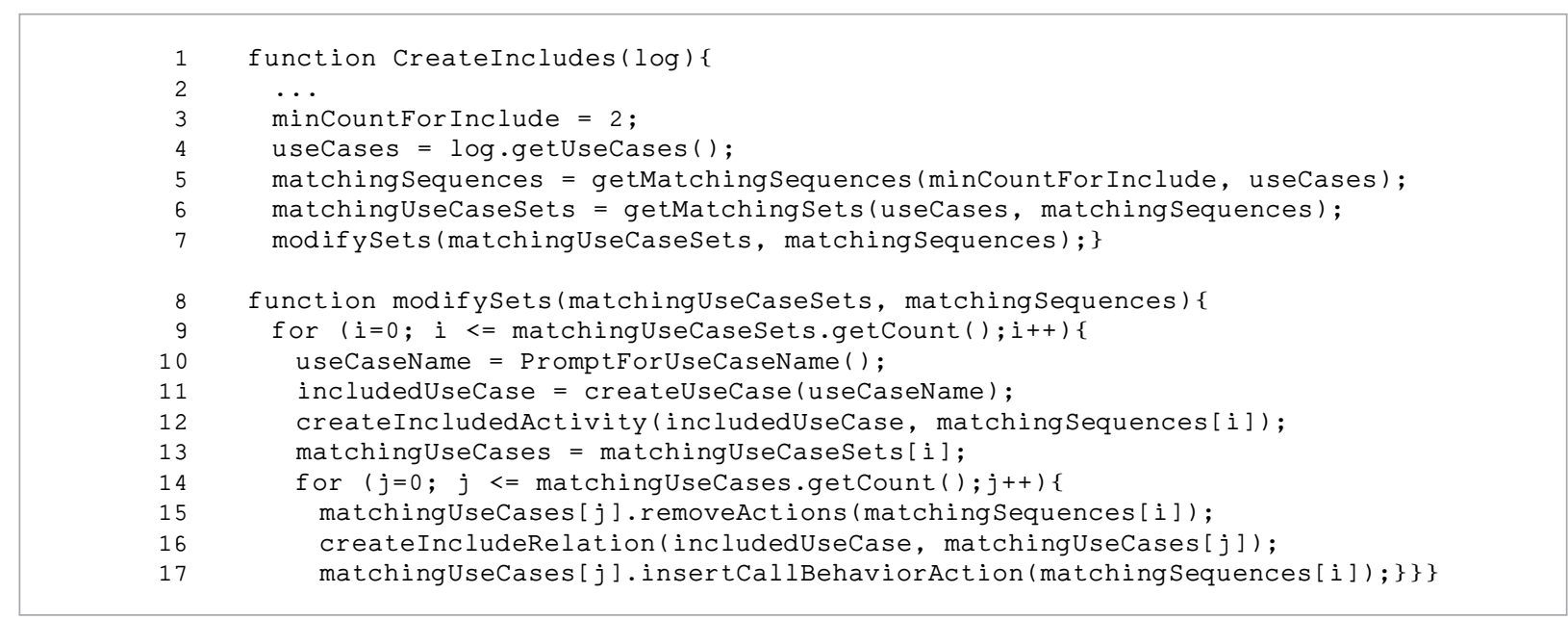

file contains one use case diagram, containing use cases, actors and their relation, and activity diagrams one for each use case.

\section{Implementation of Usage Recording and Diagram Generation Tool}

For implementing the proposed method, a tool capable of recording user actions in a website, extracting HTML code information, and transforming all gathered data into UML use case model was developed. The tool WEB2UML was implemented in a form of a plugin for Google Chrome browser. The main view of WEB2UML tool user interface is presented in Figure 6.

User activity recording implementation in WEB2UML is based on the procedure, which starts with user providing the name for the role he undertakes in the web system and the process he is going to record. Afterwards, the user performs necessary actions for fulfilling the process. The process with the same name can be recorded several times, thus demonstrating all possible alternative scenarios of process execution. User can record other processes, until he is satisfied with the recorded scope. After finishing the recording, user can export the recorded information in a form of JSON (Figure 7). The recorded information in JSON format can be shared between users, later exported and imported for further use.

\section{Figure 6}

The implemented WEB2UML plugin

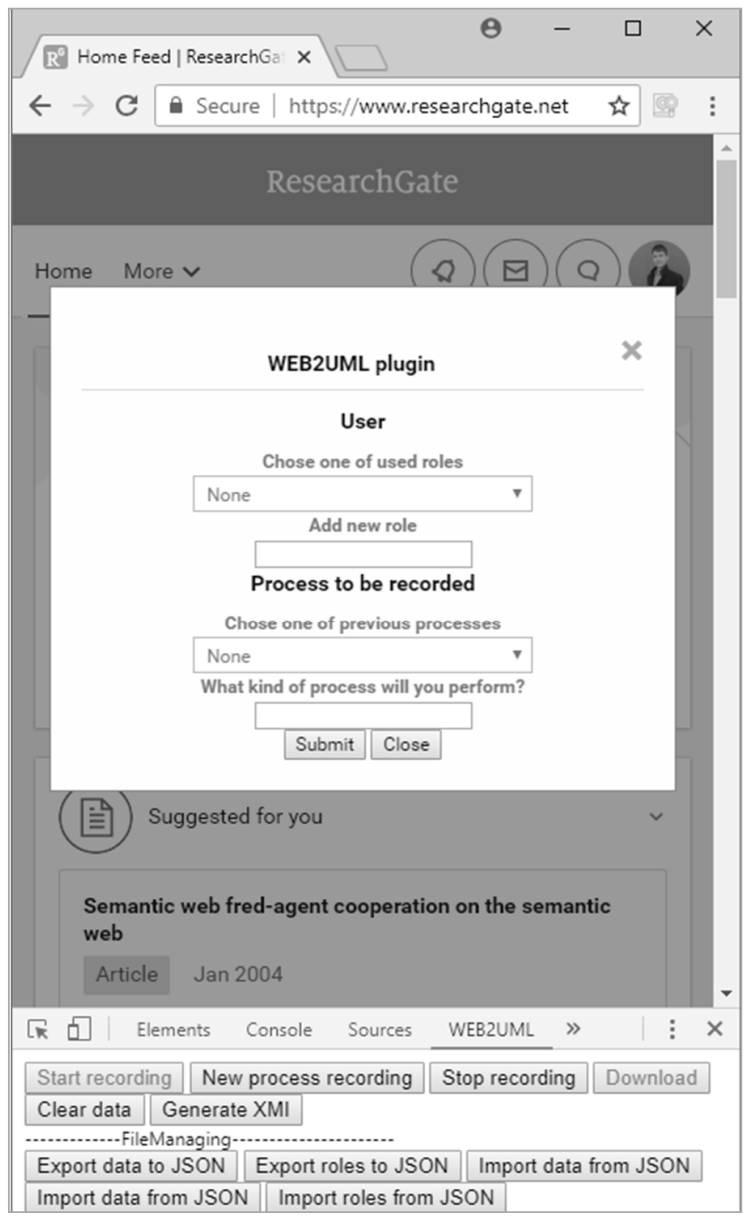


Figure 7

Fragment of JSON file for activity recording

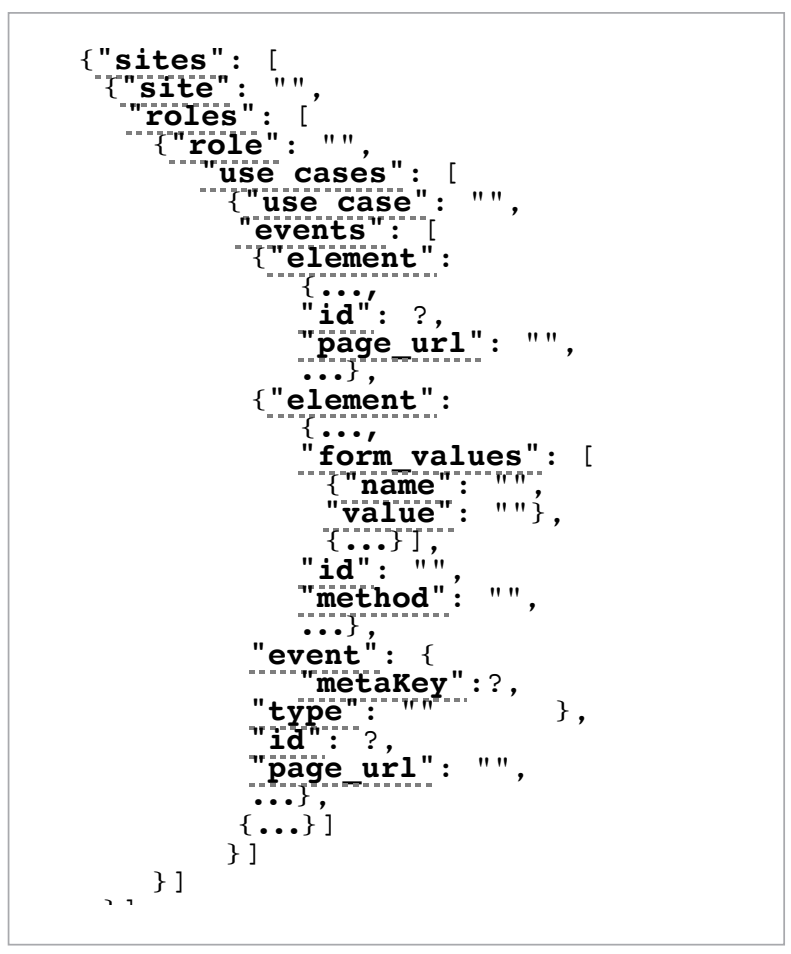

The functionality of XMI generation in WEB2UML tool covers both analysis of recorded data for creating diagrams with required include, extend, actor generalization relations and transforming the analysis results into XMI file. The XMI file generated by WEB2UML tool is compatible with CASE tool MagicDraw UML and can be opened in this tool for further analysis and modification.

The implemented WEB2UML tool was tested by generating several use case models for web applications, which are already documented and comparing the reversed UML diagrams to the ones already provided in websites documentation. For the testing, the graduate computer science students' final projects were used, where web systems were implemented and documented using UML diagrams, including use case and activity diagrams. WEB2UML was able to generate use case models for all websites used in testing. In general, reverse engineering was successful, as most of the diagram elements in reversed diagrams corresponded to the diagram elements in websites documentation. However, it is worth noting that WEB2UML was not able to fully evaluate and correctly record some actions related to iframe elements in HTML as well as JavaScript intensive website functionality. In the future, recording functionality of WEB2UML must be improved, to ensure full recording of website usage data. Nonetheless, current range of capabilities of WEB2UML is sufficient for demonstrating the functionality of generating UML diagrams based on recorded data.

\section{The Experiment}

The experiment was performed to assess WEB2UML tools' ability to reverse engineer UML models from selected websites and the quality of reversed models. The experiment was carried out in two phases. First, two use case models for selected websites were reversed using the WEB2UML tool. Then, the generated models were analyzed and evaluated by field experts in UML, having various experience in UML modelling or certified by OMG.

As the assessment of WEB2UML reverse engineered models requires expert knowledge, the anonymous questionnaire was created and sent to the list of known experts working both in academic and in business sectors. The evaluation of the models is a time-consuming task: experts not only need to analyze the provided UML use case diagram and each use cases' activity diagrams, but also to familiarize with systems functionality and usage scenarios and then finally evaluate each model based on the provided aspects. With the intention to collect as many responses as possible, only two websites were chosen for reverse engineering and evaluation. In total, 18 invitations to fill out the questionnaire were extended, and 13 experts completed the questionnaire.

\section{The Generated Use Case Models}

UML use case models for selected websites were generated based on system usage scenarios, which were created to demonstrate method capabilities. The scenarios were developed to include two types of generalizations between actors (partial and full, see Figure 2, line 9 and line 16). Scenarios also cover situations where include and extend relations between use cases should be detected. 
The first model was reversed for Moodle website https:// moodle.if.ktu.lt. Two roles were recorded: student and teacher. The tasks, that might be familiar to the experts, were chosen for clarity of the generated models. Student logged in and performed some tasks - downloaded course material, completed a test composed of 10 questions, and filled out a feedback form. Teacher logged in as well, then downloaded course material, and completed the same test. Additionally, teacher added a new file and removed an old one from the course, as well as edited a students' registration date.

The reversed use case diagram for Moodle website is presented in Figure 8. The tool detected generalization between student and teacher actors: their sets of use cases had intersection including processes of logging in, downloading file and performing test. Therefore, additional actor was created (course user) and associated with use cases from the intersection set.

\section{Figure 8}

Use case diagram reversed engineered from Moodle website

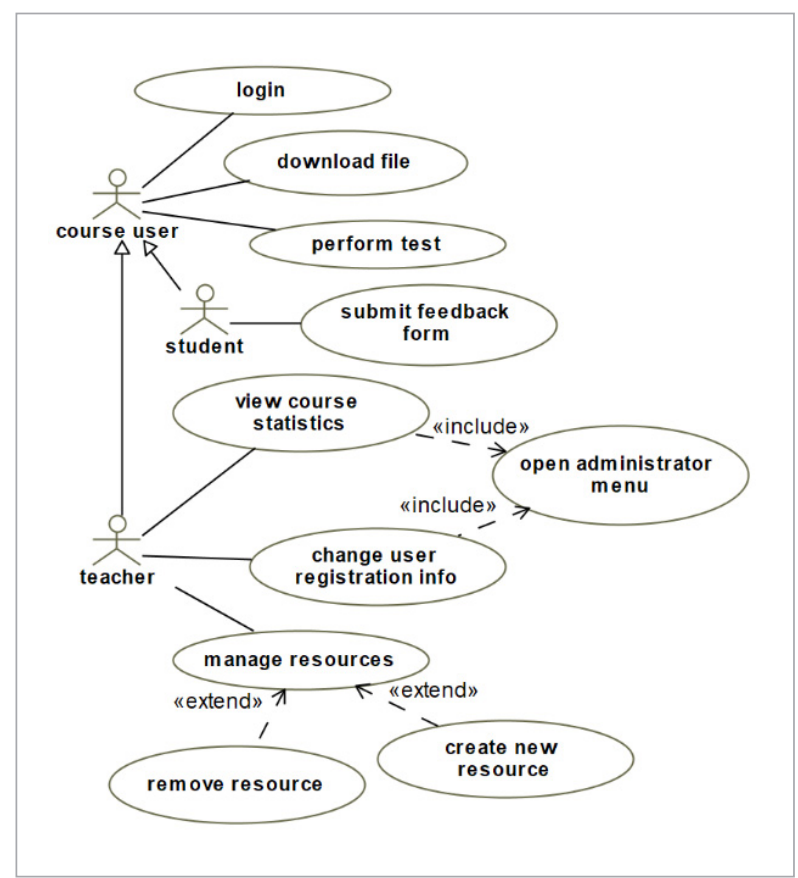

Both include and extend relations were properly detected in reversed use case model. Use cases of viewing course statistics and changing users' registration information included the set of actions for opening administrator menu. Therefore, this action set was trans- ferred into included use case and connected to including use cases by include relations. For the use case of managing resources, three alternative scenarios were recorded and combined into a single use case. For this process, two additional extending use cases were created (remove resource and create new resource).

Activity diagrams were generated for every use case in reversed model for Moodle website. An example of activity diagram for login process is presented in Figure 9. Each activity diagram has two swimlanes, representing the user and the system under analysis. Actions are named using the information extracted from websites HTML code where possible. Some system actions (e.g. process form data) were named according to the expected typical reactions of the system.

\section{Figure 9}

Activity diagram for the recorded process of logging in to Moodle website

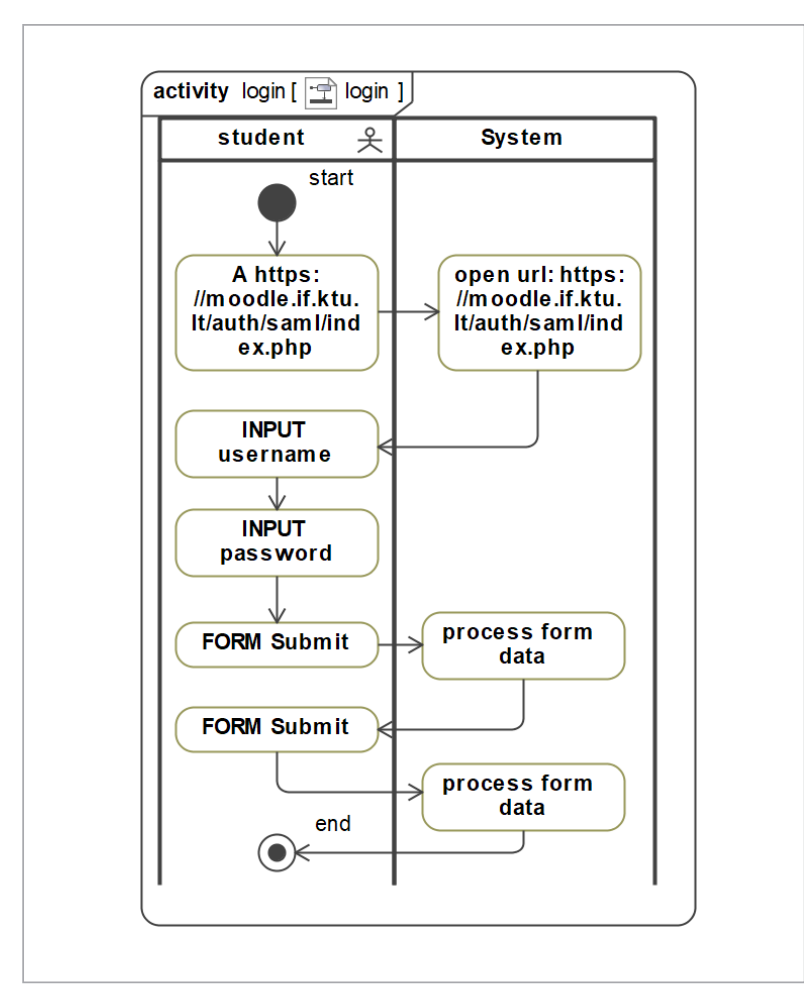

Activity diagram for use case of viewing the statistics of the course is presented in Figure 10. Include relation was correctly represented in generated activity diagram - reference to activity for use case of opening 
administration menu was inserted in the beginning of the generated diagram. Reference to included use cases' activity diagram is indicated by the colon before action's name and can be additionally represented using the rake icon inside the action node.

\section{Figure 10}

Activity diagram for the recorded process of viewing course statistics in Moodle website

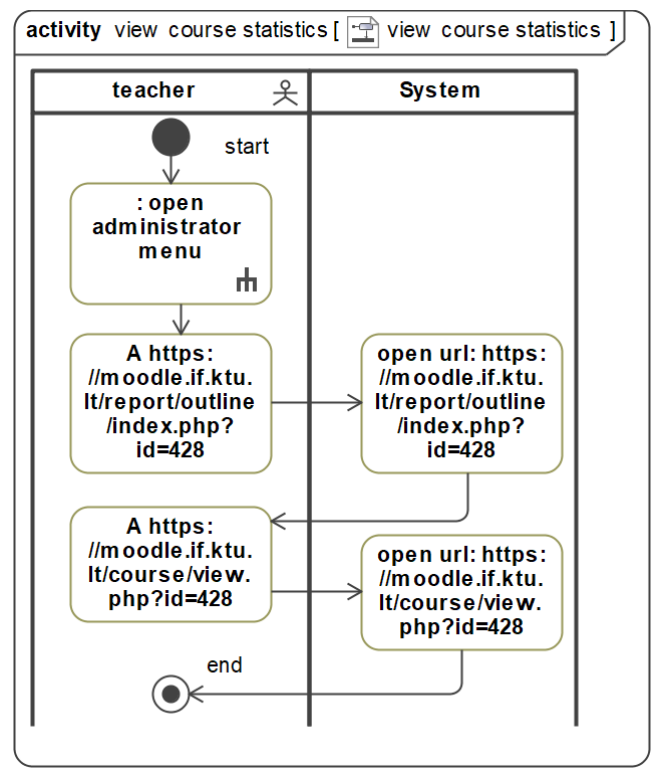

For reversing the second use case model, a usage of ResearchGate website (https://researchgate.net) was recorded. Two user roles were defined: one for the author and the other for the reader. Reader logged in, requested access to the full text of the research publication and viewed job listings. The author performed the same processes as the reader. Additionally, the author changed his profile settings, edited one research publication and removed one research publication. The author also added a new research publication (two scenarios were recorded for this process: one for just adding the publication information, and the other for adding publication information with additional actions of attaching a file).

Reverse engineered use case diagram for ResearchGate website is presented in Figure 11. In generated diagram, generalization between actors was success-

\section{Figure 11}

Use case diagram reversed engineered from ResearchGate website

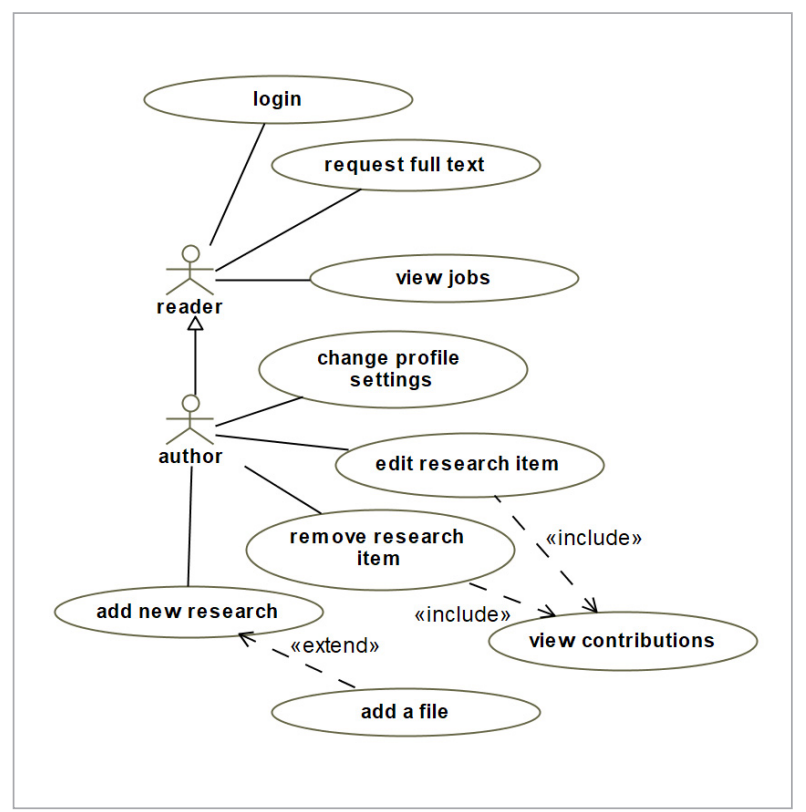

fully detected and created, as the author inherits all use cases associated with the reader. Included use case of viewing the contributions was created. This included use case was connected using include relation to use cases of research item editing and removing. Extending use case was also created for the use case of adding the new research publication.

The activity diagram for the use case of adding new research publication is presented in Figure 12. As two scenarios were recorded for this use case, the tool combined them into one activity diagram. During the process of combining, extend relation was detected and new extending use case for adding a file was created. This was also represented in extended use cases' activity diagram in Figure 12, as it contains both decision and merge nodes and a reference (callBehaviorAction) to the extending use cases' activity diagram.

The generated models along with the predefined scenarios were provided to experts for evaluation. A questionnaire was prepared for evaluating the models. The experts answered equivalent questionnaire questions for both Moodle and ResearchGate use case models. 


\section{Figure 12}

Activity diagram for the recorded process of adding new research publication in ResearchGate website

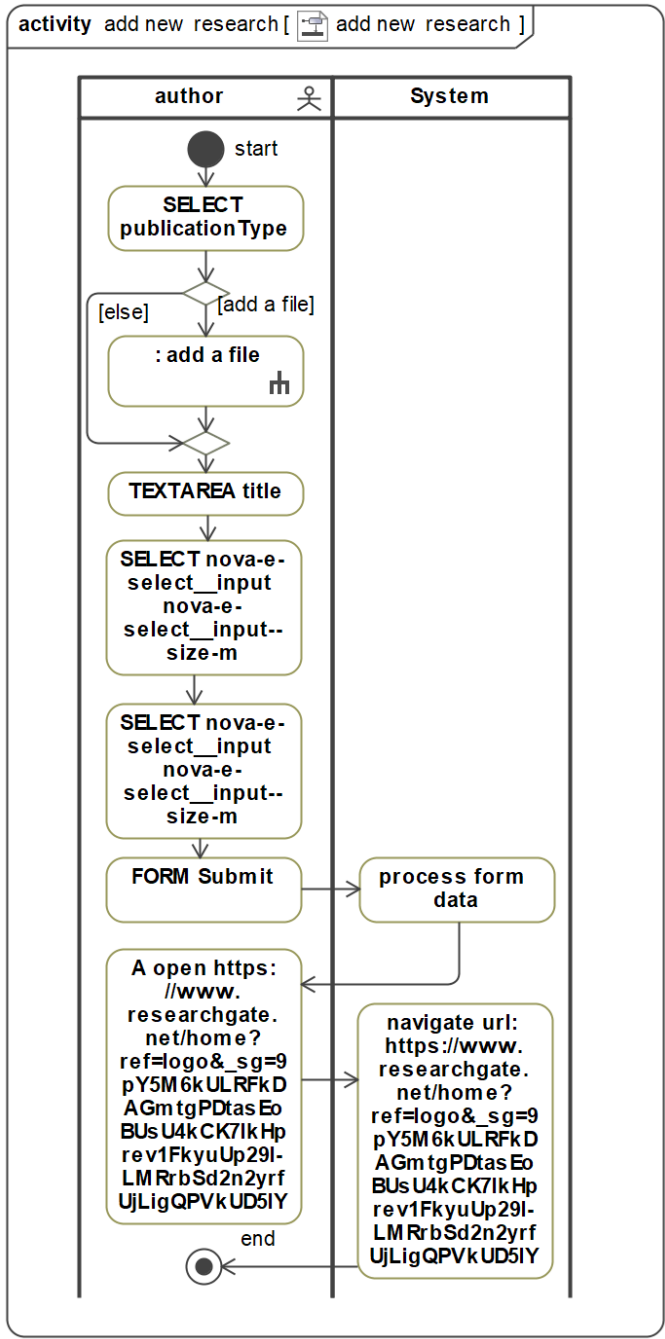

\section{The Expert Evaluation Results}

Altogether, 13 experts completed the anonymous questionnaire. Before evaluating the models, experts were asked to outline their experience in UML modelling. In total, four of the experts declared, that they are OMG certified UML Professionals: two Intermediate and two Advanced level. The declared expert experience in UML modelling varies: six of them have nine or more years of experience, two have between five and eight years of experience and five experts have between two and four years of experience.

The questions in the questionnaire were grouped according to the evaluated diagram type. Two question groups were created - one for use case diagram and the other for all activity diagrams in the model. For evaluation, experts had to choose a score from one to ten for each question. The questions for both groups are presented in Table 1. Questions in questionnaire were designed to evaluate syntax and semantics of generated UML use case model. Experts were asked to evaluate use case diagrams by assessing overall quality of generated use case diagram (question 1) and elements of the diagram as well as their relations (question 2-4). Experts also had to evaluate the naming of use case diagram elements (question 5). Activity diagrams were evaluated by assessing the overall quality of the scenarios' representations (question 6). Experts were asked to assess the consistency between use case and activity diagrams: how extend and include relations correspond to specific combinations of elements in activity diagrams (question 7). In addition, the quality of generated names for activity diagram elements was assessed (question 8). All questions in the questionnaire were of equal weight.

\section{Table 1}

The list of questions in model evaluation questionnaire

\section{Use case diagram evaluation}

1. Rate the quality of the generated use case diagram for given system scenarios

2. Rate the quality of the generated generalization relations between actors

3. Rate the quality of the generated extend relations between use cases

4. Rate the quality of the generated include relations between use cases

5. Rate the quality of use case diagram element names

\section{Activity diagrams evaluation}

6. Rate the quality of the generated activity diagrams for given system scenarios

7. Rate the quality of include and extend relations' representation in activity diagrams

8. Rate the quality of activity diagrams element names 
Out of the collected responses, the arithmetic means of each expert evaluation scores for Moodle and ResearchGate use case models were calculated (Figure 13). As we can see, the use case model for Moodle website was evaluated more favorably with mean score of 8.5 and a much smaller distribution between expert given scores. ResearchGate evaluation score is more varied: the mean score of 8.3 is fairly similar, but with larger distribution of given scores. Larger distribution of evaluation scores for ResearchGate model may be caused by insufficient WEB2UML capabilities of recording. ResearchGate website employs HTML code obfuscation and implements a wide range of JavaScript functionality, which is why the recording tool was not able to correctly record some of the actions performed by the user.

\section{Figure 13}

The boxplot diagram for model evaluation scores

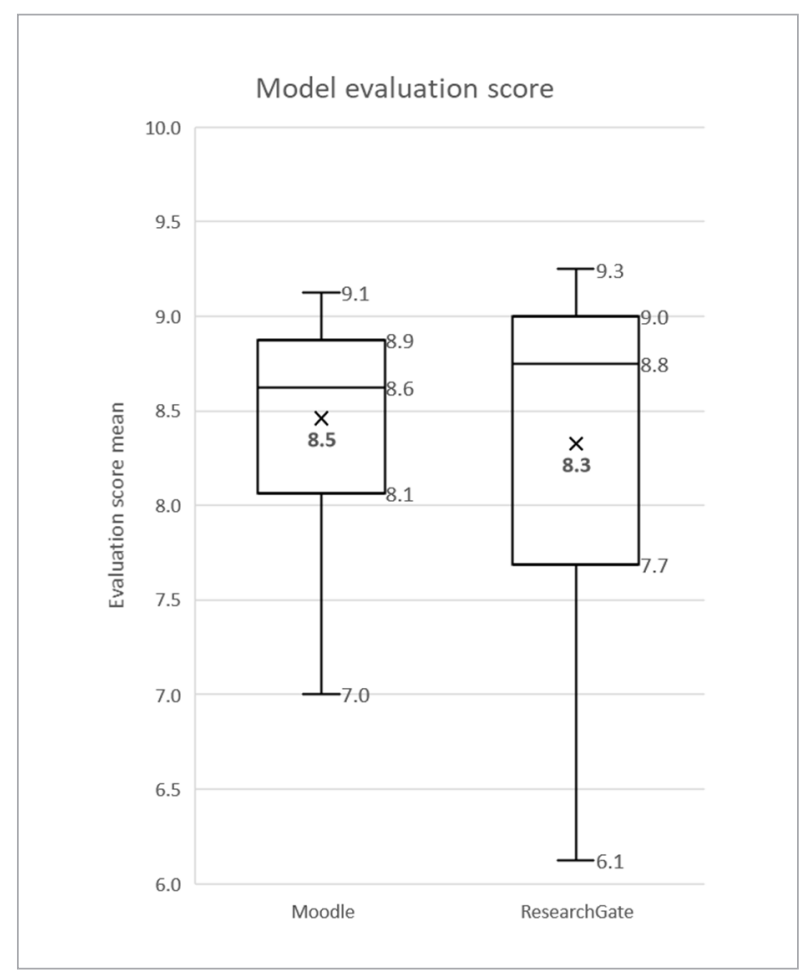

Additionally, evaluation score means for each question about the models were calculated (Figure 14). The results are quite similar among the questions, only questions about activity diagrams and activity diagrams' elements naming are clear outliers.
The quality of the naming in activity diagram was evaluated in lowest scores (4.8 for ResearchGate and 4.5 for Moodle). The problems with action names might have also be the cause of the poor evaluation of activity diagrams in general. On the other hand, other aspects of generated models received quite encouraging evaluation. Use case diagrams for both models were evaluated very positively (8.5 for ResearchGate and 9.0 for Moodle). Relation detection capabilities of WEB2UML were also favorably evaluated, as the score means for the questions about generalization, include and extend relations was above 9.1 for both models.

\section{Figure 14}

The mean scores for each question of model evaluation

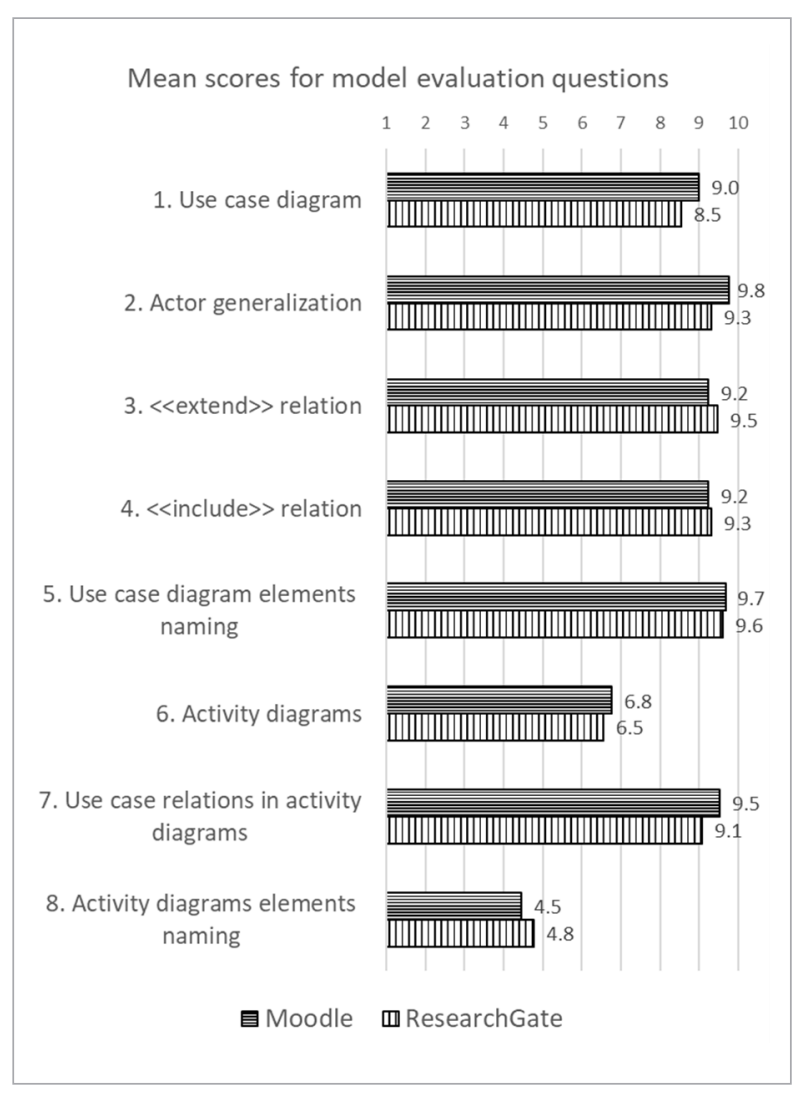

\section{Discussion}

The experimental results indicate that WEB2UML tool is able to reverse engineer the use case model for a website. The tool generates use case diagram with sce- 
narios for each use case in a form of activity diagrams. The resulting use case diagram is similar to reversed use case diagrams presented in $[6,9,16]$, as it encompasses the main elements of use case diagram: use cases, include and extend relations. Our tool also incorporates actors and actor generalizations into the use case diagram, which are only present in [9] generated use case diagram. On the other hand, our tool does not detect use case generalizations, while approach in [6] does. The main difference between our method and approaches in $[6,9,16]$ is that our method does not require access to internal source code of the system. The other significant difference is that our approach also incorporates activity diagram generation for each use case. Other approaches either do not generate any diagrams for use case scenario representation $([6,16])$ or use sequence diagrams $([9])$. The methods not requiring access to source code, like [11, 19], cannot be directly compared to our approach, as they require additional specifications for diagram generation. The contents and quality of diagrams generated in $[11,19]$ directly depends on the quality of the prepared additional specifications.

The results of expert evaluation of diagrams generated by WEB2UML tool are promising, as experts positively evaluated the generated use case models (the mean of all expert evaluation scores is 8.4). Both Moodle and ResearchGate models were evaluated fairly similarly among all the questions in questionnaire. The drawbacks of usage recording tool caused slightly lower evaluation of the model for ResearchGate website. Another problem for both models is action naming in activity diagrams. Current version of WEB2UML is not able to generate suitable names for the actions, based on the information extracted from websites HTML code. In the future, we are going to improve the tools capabilities of generating the adequate names for actions.

\section{Conclusions}

UML diagrams are utilized during various phases of software development lifecycle, including implementation and maintenance. Unfortunately, the task of maintaining the documentation up to date is costly, so is the process of manual recovering of documentation for legacy systems. Reverse engineering can alleviate this problem by providing means for creating visual representations of analyzed systems. In this paper, the method for reverse engineering UML use case model for websites is presented. The proposed solution is implemented as a Google Chrome plugin named WEB2UML and is able to generate UML use case and activity diagrams describing interactions between the user and the system. These diagrams are generated in XMI format, compatible with MagicDraw UML CASE tool. WEB2UML tool is able to create required include and extend relations between use cases as well as generalizations between actors in use case diagram. It also enables combining of user recorded processes for the same use case into activity diagrams with decision nodes.

During experimental evaluation of the tool, two UML use case models were reverse engineered: one for moodle.if.ktu.lt website and another for researchgate. net website. The WEB2UML tool was able to reverse engineer UML models for corresponding websites according to the usage scenarios, which were created to demonstrate method capabilities. Both reversed use case diagrams encompassed include and extend relations and generalizations between actors. Activity diagrams were generated for every use case in the model and alternative usage scenarios were successfully combined into activity diagrams. The main drawback of reverse engineered models was the naming of the actions in activity diagrams. The information for action names was extracted from websites publicly available HTML code, but this process did not produce satisfactory result and should be further improved.

The quality of generated models was evaluated using a questionnaire for UML modelling experts. In total, 13 experts participated in the experiment by evaluating the generated models and completing the questionnaire. The results of expert evaluation are encouraging, as experts positively evaluated both reversed use case models, with exception of naming in activity diagrams, which received quite negative evaluation. Relations between elements, both in use case and activity diagrams received the highest evaluation scores. Altogether, the mean of expert evaluation scores was 8.4 in a scale of ten.

In the future we are planning to improve the method and its implementation for generating more adequate action names in activity diagrams. The capabilities of recording user actions of WEB2UML tool should also be improved, which would significantly increase applicability of the proposed method. 


\section{References}

1. Aabidi, M. H., Mahi, B. El, Baidada, C., Jakimi, A., Ammar, $\mathrm{H}$. Benefits of Reverse Engineering Technologies in Software Development Makerspace. ITM Web of Conferences, 2017, 13, 01028. https://doi.org/10.1051/ itmconf/20171301028

2. Alalfi, M. H., Cordy, J. R., Dean, T. R. Automated Reverse Engineering of UML Sequence Diagrams for Dynamic Web Applications. International Conference on Software Testing, Verification and Validation Workshops, 2009, 287-294. https://doi.org/10.1109/ICSTW.2009.8 https://doi.org/10.1109/ICSTW.2009.8

3. Bolloju, N., Sun, S. X. Benefits of Supplementing Use Case Narratives with Activity Diagrams-An Exploratory Study. Journal of Systems and Software, 2012, 85(9), 2182-2191. https://doi.org/10.1016/j.jss.2012.04.076

4. Bruneliere, H., Cabot, J., Dupé, G., Madiot, F. MoDisco: A Model Driven Reverse Engineering Framework. Information and Software Technology, 2014, 56(8), 10121032. https://doi.org/10.1016/j.infsof.2014.04.007

5. Chikofsky, E., Cross, J. Reverse Engineering and Design Recovery: A Taxonomy. IEEE Software, 1990, 7(1), 1317. https://doi.org/10.1109/52.43044

6. Claudia, P., Liliana, M., Liliana, F. Recovering Use Case Diagrams from Object Oriented Code: An MDA-based Approach. Eighth International Conference on Information Technology: New Generations (ITNG), Las Vegas, 2011, 737-742. https://doi.org/10.1109/ITNG.2011.130

7. da Silva, A. R. Model-Driven Engineering: A Survey Supported by the Unified Conceptual Model. Computer Languages, Systems \& Structures, 2015, 43, 139-155. https://doi.org/10.1016/j.cl.2015.06.001

8. Decker, M. J., Swartz, K., Collard, M. L., Maletic, J. I. A Tool for Efficiently Reverse Engineering Accurate UML Class Diagrams. IEEE International Conference on Software Maintenance and Evolution (ICSME), 2016, 607-609. https://doi.org/10.1109/ICSME.2016.37

9. Di Lucca, G. A., Fasolino, A. R., Tramontana, P. Reverse Engineering Web Applications: The WARE Approach. Journal of Software Maintenance and Evolution: Research and Practice, 2004, 16(1-2), 71-101. https://doi. org/10.1002/smr.281

10. Dick, J., Hull, E., Jackson, K. Requirements Engineering. Springer, 2017. https://doi.org/10.1007/978-3-31961073-3

11. El-Attar, M., Miller, J. Producing robust Use Case Diagrams via Reverse Engineering of Use Case Descrip- tions. Software \& Systems Modeling, 2008, 7(1), 67-83. https://doi.org/10.1007/s10270-006-0039-3

12. Fernández-Sáez, A. M., Chaudron, M. R., Genero, M. An Industrial Case Study on the Use of UML in Software Maintenance and Its Perceived Benefits and Hurdles. Empirical Software Engineering, 2018, 1-65. https:// doi.org/10.1007/s10664-018-9599-4

13. Jiang, J., Koskinen, J., Ruokonen, A., Systa, T. Constructing Usage Scenarios for API Redocumentation. 15th IEEE International Conference on Program Comprehension, 2007, 259-264. https://doi.org/10.1109/ICPC.2007.16

14. Kaur, H., Ahamad, S., Verma, G. N. Elements of Legacy Program Complexity. International Journal of Research in Engineering and Technology, 2015, 4(3), 501505. https://doi.org/10.15623/ijret.2015.0403085

15. Larman, C. Applying UML and Patterns: An Introduction to Object Oriented Analysis and Design and Iterative Development. Dorling Kindersley Pvt. Ltd., 2008.

16. Li, Q., Hu, S., Chen, P., Wu, L., Chen, W. Discovering and Mining Use Case Model in Reverse Engineering. Fourth International Conference on Fuzzy Systems and Knowledge Discovery, Haikou, China, 2007, 4, 431-436. https://doi.org/10.1109/FSKD.2007.255

17. Martin, R., Archer, L. Reverse Engineering of Web Applications: A Technical Review. University of Liverpool, 2007.

18. Martins, L. C. G., Garcia, R. E., Marçal, I. Using Information Visualization to Comprehend User Interface Layer: An Application to Web-Based Systems. Proceedings of the XVI Brazilian Symposium on Human Factors in Computing Systems, 2017, 49. https://doi. org/10.1145/3160504.3160558

19. Mohammad, I. M., Rafa, E. A.-Q. An Approach to Derive the Use Case Diagrams from an Event Table. SEPADS'09 Proceedings of the 8th WSEAS International Conference on Software Engineering, Parallel and Distributed Systems, Cambridge, 2009, 33-38.

20. Morgado, I. C., Paiva, A. C., Faria, J. P. Dynamic Reverse Engineering of Graphical User Interfaces. International Journal on Advances in Software, 2012, 5(3-4), 224-236.

21. Murugesan, S. Web Application Development: Challenges and the Role of Web Engineering. Web Engineering: Modelling and Implementing Web Applications, Springer-Verlag London, 2008, 7-32. https://doi. org/10.1007/978-1-84628-923-1_2

22. Noda, K., Kobayashi, T., Toda, T., Atsumi, N. Identifying Core Objects for Trace Summarization Using Reference 
Relations and Access Analysis. IEEE 41st Annual Computer Software and Applications Conference (COMPSAC), Turin, 2017, 13-22. https://doi.org/10.1109/ COMPSAC.2017.142

23. NoMagic, Inc. MagicDrawUML. https://www.nomagic. com/products/magicdraw. Accessed on July 10, 2018.

24. Object Management Group. UML 2.5 Specification. http://www.omg.org/spec/UML/2.5/PDF. Accessed on March 1, 2015.

25. Osman, H., Chaudron, M. R. Correctness and Completeness of CASE Tools in Reverse Engineering Source Code into UML Model. GSTF Journal on Computing, 2012, 2(1), 193-201. http://dx.doi.org/10.5176_20102283_2.1.150

26. Prause, C. R., Durdik, Z. Architectural Design and Documentation: Waste in Agile Development? IEEE International Conference on Software and System Process (ICSSP), 2012, 130-134. https://doi.org/10.1109/ ICSSP.2012.6225956

27. Raibulet, C., Fontana, F. A., Zanoni, M. Model-Driven Reverse Engineering Approaches: A Systematic Literature Review. IEEE Access, 2017, 5, 14516-14542.https:// doi.org/10.1109/ACCESS.2017.2733518
28. Schmidt, D. Model-Driven Engineering. Computer, 2006, 39(2), 25-31.

29. Sparx Systems Pty Ltd. Enterprise Architect. http:// www.sparxsystems.com.au/products/ea/index.html. Accessed on July 10, 2018.

30. Tiwari, S., Gupta, A. A Systematic Literature Review of Use Case Specifications Research. Information and Software Technology, 2015, 67, 128-158. https://doi. org/10.1016/j.infsof.2015.06.004

31. Visual Paradigm. https://www.visual-paradigm.com/. Accessed on July 10, 2018.

32. Yang, J., Lee, Y., Gandhi, D., Valli, S. G. Synchronized UML Diagrams for Object-Oriented Program Comprehension. 12th International Conference on Computer Science and Education (ICCSE), Houston, TX, USA, 2017, 12-17. https://doi.org/10.1109/ ICCSE.2017.8085455

33. Ziadi, T., da Silva, M. A. A., Hillah, L. M., Ziane, M. A Fully Dynamic Approach to the Reverse Engineering of UML Sequence Diagrams. 16th IEEE International Conference on Engineering of Complex Computer Systems, ICECCS, Las Vegas, United States, 2011, 107-116. https://doi.org/10.1109/ICECCS.2011.18 